\title{
ASPECTOS ESTRUTURAIS DE ESFERAS DE QUITOSANA/PVA RETICULADAS COM GLUTARALDEÍDO SUBMETIDAS A DIFERENTES TRATAMENTOS TÉRMICOS
}

\author{
Thamy G. P. Sobreira ${ }^{a, c}$, Lindomar A. da Silva ${ }^{b}$, Frederico D. de Menezes ${ }^{b}$, Elvis J. França ${ }^{c}$ e Kátia Aparecida da Silva \\ Aquino $^{\mathrm{a}, *, \mathbb{D}}$ \\ aDepartamento de Energia Nuclear, Universidade Federal de Pernambuco, 50740-540 Recife - PE, Brasil \\ 'Instituto Federal de Pernambuco, 50740-545 Recife - PE, Brasil \\ ${ }^{\circ}$ Centro Regional de Ciências Nucleares do Nordeste, 50740-545 Recife - PE, Brasil
}

Recebido em 19/05/2020; aceito em 30/06/2020; publicado na web em 20/08/2020

\begin{abstract}
STRUCTURAL ASPECTS OF CHITOSAN/PVA SPHERES CROSS-LINKED WITH GLUTARALDEHYDE AND SUBMITTED TO DIFFERENT THERMAL TREATMENTS. Chitosan is a biopolymer derived from chitin and the most abundant polysaccharide in nature after cellulose. This biopolymer has been applied in different areas due its biodegradability and biocompatibility properties. Modifications of chitosan structure by cross-linking chemical agents can alter its physical, chemical, and morphological characteristics. The present work aimed at studying the structural characteristics of chitosan/polyvinyl alcohol (PVA) spheres cross-linked with glutaraldehyde submitted to oven-drying and freeze-drying processes. The thermal-treated of spheres and chitosan (control sample) were analyzed by XRD, SEM and FTIR techniques. Multivariate statistical analysis, PCA, was applied to samples IR spectra. According to results, the freeze-dried spheres showed a smaller crystal size $(6.032 \mathrm{~nm})$, while the oven-dried spheres presented a smaller crystalline phase (3.49\%). MEV images have encompassed the different surfaces of the manufactured spheres as a result of drying methods. Slight chemical changes were observed in the IR spectra from the cross-linked spheres. In addition, PCA has revealed different clusters referred to the structural modifications. Therefore, the increasing of openness and receptivity for chemical reactions, as well as the adsorption capacity, could enable different applications of the chitosan, mainly the adsorption properties to remove residues and for water treatment.
\end{abstract}

Keywords: nanospheres; cross-linking; chemometrics; drying.

\section{INTRODUÇÃO}

A quitosana, por ser derivada da quitina, é considerada o segundo biopolímero mais abundante na natureza depois da celulose. Essa substância pode ser encontrada em fungos, insetos e artrópodes, ${ }^{1,2}$ sendo extraída das carapaças de caranguejos, camarão e lagosta, resíduos da produção industrial. ${ }^{3}$ Adicionalmente, fungos como Rhizopus oryzae ou Mucor rouxii são alternativas para produção de quitosana às limitações da indústria pesqueira devido à produção sazonal, local e de custo elevado..$^{1,4}$

A obtenção da quitosana ocorre a partir da desacetilação da quitina, com a retirada do grupo acetila por meio de tratamento químico com hidróxido de sódio, resultando em estruturas repetidas de $\beta$-(1-4) 2-acetamido-deoxi-D-glicose ( $\mathrm{N}$-acetilglicosamina), ou seja, D-glucosamina. ${ }^{2,3}$ As concentrações do hidróxido de sódio, tempo e temperatura de reação para desacetilação influenciam o grau de pureza e as propriedades da quitosana. Os grupos funcionais restantes como hidroxila $(-\mathrm{OH})$ e amina $\left(-\mathrm{NH}_{2}\right)$ tornam esse biopolímero versátil para diversas aplicações. ${ }^{3,5}$

Por outro lado, a quitosana, associada a outros materiais, pode ser aplicada na odontologia, por meio da produção de scaffolds como elemento de tratamento ou para tratamento de canais, puro ou em associação com peróxido de carbamida, com o transportador de cimento. ${ }^{6-8}$ Ainda na odontologia, é possível utilizar cimento de ionômero com acréscimo de quitosana como alternativa à amálgama e resina. ${ }^{3,9}$ Além disso, sua aplicação se estende à área médica como adjuvante de vacinas ou, na área ambiental, como adsorvente de poluentes em suspensão. ${ }^{10,11}$ Em outras aplicações, por exemplo, na área da genética, pode ser realizada a síntese de nanopartículas

*e-mail: aquino@ufpe.br de ouro e quitosana para explorar alternativas de transferência de DNA.

As características físicas, químicas e morfológicas determinam as aplicações possíveis da quitosana, de forma que a quitosana modificada pela introdução de grupos hidrofílicos, por exemplo, pode ter suas propriedades de superfície melhoradas. ${ }^{12}$ Essas modificações podem ocorrer por meio de reticulação na forma de filmes de quitosana com glicerol, alterando sua flexibilidade e os efeitos hidrofílicos, ou de filmes com adição de solução de cério para uso de curativos antimicrobianos. ${ }^{3,13,14}$ Além de filmes, modificações na estrutura da quitosana pode ocorrer pela reticulação em diferentes formas, como esferas e microesferas, aplicada na área ambiental para adsorção de metais tóxicos, como Cr e radionuclídeos como Th, U e Cs, por exemplo. ${ }^{5,15}$ Essa reticulação pode ser realizada por agentes químicos como etileno glicol diglicidílico éter (EGDE), epicloridrina, glutaraldeído e tripolifosfato (TPP), seguido de secagem. . $^{5,15}$

Utilizar agentes de reticulação possibilita também o uso da quitosana na área farmacêutica, por meio da preparação de esferas, para controle de liberação de medicação. Assim, tais esferas podem configurar uma alternativa a polímeros sintéticos, proporcionando a fabricação de materiais biodegradáveis como celulose e quitosana, que juntamente com outros polímeros como o acetato de polivinila (PVA), por exemplo, fornece um material com biocompatibilidade com a vantagem da combinação das suas propriedades físicas. ${ }^{16-18}$ Tratamentos térmicos durante a produção desses materiais permitem alterar estados mecânicos e químicos, modificando o material para aplicações específicas. ${ }^{19-21}$

Diante do exposto, o presente trabalho tem como objetivo estudar características estruturais da quitosana e de esferas de quitosana/PVA reticuladas com glutaraldeído submetidas a tratamentos térmicos por liofilização e secagem em estufa de circulação forçada de ar. 
As análises estruturais foram realizadas pelas técnicas de Difração de Raios-X (DRX), Microscopia Eletrônica de Varredura (MEV) e Espectroscopia no Infravermelho com Transformada de Fourier (FTIR). Estatística multivariada por Análises dos Componentes Principais (PCA) foi aplicada aos espectros de FTIR obtidos para avaliar possíveis diferenças entre as esferas produzidas.

\section{PARTE EXPERIMENTAL}

\section{Fabricação das esferas de quitosana/PVA}

A quitosana utilizada no trabalho foi obtida comercialmente (Polymar ${ }^{\circledR}$ ) com densidade aparente de $1,8 \mathrm{~g} \mathrm{~L}^{-1}, \mathrm{pH}$ de 8,4 e grau de desacetilação de $85 \%$. Todos os reagentes utilizados foram de grau analítico e a vidraria foi descontaminada com solução de detergente científico (10\%) e ácido nítrico destilado (10\%), seguido de lavagem com água ultrapura (resistividade de $18 \mathrm{M} \Omega \mathrm{cm}^{-1}$ ).

Para a produção das esferas de quitosana/PVA foram utilizados ácido acético - $\mathrm{CH}_{3} \mathrm{COOH}(\sim 99 \%)$, acetato de polivinila - PVA, hidróxido de sódio - $\mathrm{NaOH}$, ácido bórico $-\mathrm{H}_{3} \mathrm{BO}_{3}$ e glutaraldeído $(25 \%) \cdot{ }^{15-17}$ As etapas de produção foram sumarizadas no fluxograma (Figura 1). Inicialmente, a dissolução da quitosana em pó (5\%) foi realizada em solução de ácido acético $(10 \%)$ durante $24 \mathrm{~h}$, empregando-se água destilada. Simultaneamente, uma solução de PVA (5\%) em água destilada foi obtida com auxílio de misturador magnético aquecido em potência intermediária, sendo armazenada por $24 \mathrm{~h}$. Foi preparada a solução de quitosana $(5 \%)$ e PVA $(5 \%)$, na proporção de 1:1. Inicialmente a quitosana (5\%) foi transferida para Becker de $50 \mathrm{~mL}$, então foi adicionada a solução de PVA (5\%) e misturadas com auxílio de bastão de vidro por 60 segundos. Na solução quitosana/ PVA foi gotejada, com auxílio de pipeta descartável a cerca de $10 \mathrm{~cm}$ de distância, em solução de ácido bórico (2\%) e hidróxido de sódio $(2,5 \%)$ com o auxílio do misturador magnético e o volume foi completado para $600 \mathrm{~mL}$. As esferas permaneceram submersas durante $24 \mathrm{~h}$ em temperatura ambiente. A imersão em solução de $\mathrm{NaOH}$ a $5 \%$ resultou na coagulação da quitosana na superfície das esferas, tornando-as autossuportadas, enquanto o ácido bórico facilitou a coagulação do PVA junto à quitosana.

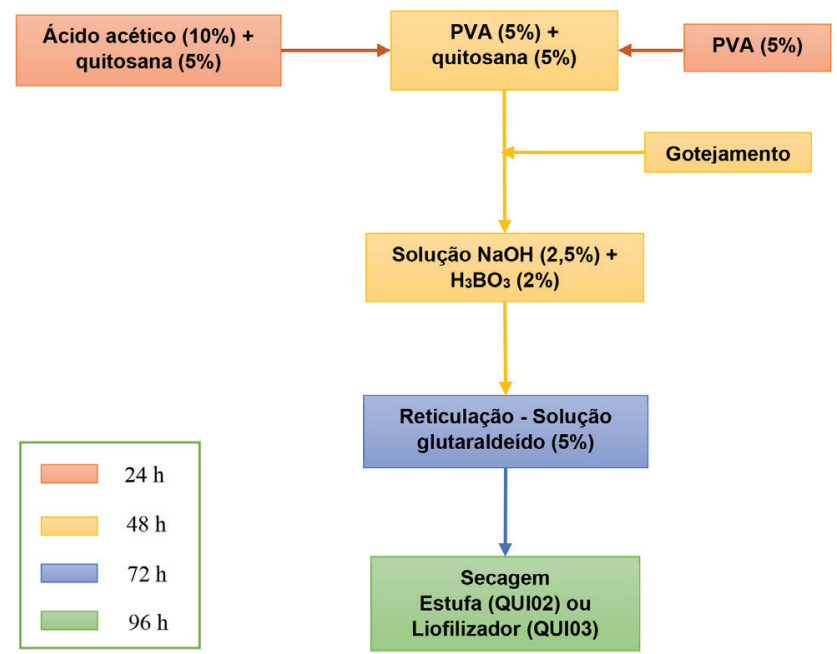

Figura 1. Fluxograma das etapas da fabricação de esferas de quitosana/PVA com tratamento térmico em estufa e em liofilizador

As esferas gotejadas apresentaram transparência, formato próximo ao esférico, sem formação de cauda. Após $72 \mathrm{~h}$ de fortalecimento das esferas coaguladas, realizou-se a lavagem do material obtido com água destilada, seguida pela sua reticulação por meio da imersão em solução de $400 \mathrm{~mL}$ de glutaraldeído (5\%) por $24 \mathrm{~h}$, seguida de lavagem com água destilada. No quarto dia, ou seja, 96 h após o início do processo, as esferas passaram por dois tipos de tratamento térmico: secagem em estufa a $60{ }^{\circ} \mathrm{C}$ durante $24 \mathrm{~h}$ (amostras QUI02) e congelamento seguido de secagem no liofilizador durante $24 \mathrm{~h}$ (amostra QUI03). Neste trabalho a quitosana sem tratamento será chamada de QUI01.

\section{Difração de raios-X (DRX)}

As análises das amostras de quitosana em pó sem tratamento (QUI01) e das esferas secas em estufa (QUI02) e liofilizadas (QUI03) foram realizadas por difratômetro de raios-X Bruker, modelo D8 Advance. $\mathrm{O}$ equipamento possui tubo de $\mathrm{Cu}$ com radiação de comprimento de onda de 1,54060 A. A voltagem e corrente elétrica de operação foram $40 \mathrm{kV}$ e $40 \mathrm{~mA}$, respectivamente. A taxa de varredura foi de $0,05^{\circ} \mathrm{em} 1 \mathrm{~s}\left(0,05 \mathrm{~s}^{1}\right)$ em intervalo angular de $5^{\circ}$ a $80^{\circ} .{ }^{4,22,23}$ Os difratogramas foram gerados por meio do programa de computador OriginPro $9.0^{\circledR}$.

O tamanho do cristal foi determinado pela equação de DebyeScherrer (Equação 1), 4,22,24 em que D corresponde ao tamanho do cristal; $k$ uma constante dependente do material de análise que para polímeros é considerada como 0,$9 ;{ }^{4} \mathrm{~h}$ corresponde largura à metade da altura do maior pico do difratograma; $\lambda$ equivale ao comprimento de onda da radiação da fonte $(0,154060 \mathrm{~nm})$; enquanto $\theta$ corresponde ao ângulo de Bragg do pico de interesse. Os parâmetros para cálculo do tamanho de cristal foram obtidos a partir dos difratogramas plotados.

$$
D=\frac{k \lambda}{h \cos (\theta)}
$$

A cristalinidade foi determinada pelo Método de Ruland por meio da Equação $2,{ }^{25}$ em que $C(\%)$ correspondeu à fase cristalina; $I_{p}$, à integração da região dos picos na fase cristalina e, $I_{a}$ à integração da região amorfa. Os parâmetros utilizados no cálculo foram obtidos com o auxílio do OriginPro 9.0 a partir da função integrate e da função de distribuição de probabilidade Gaussiana. ${ }^{26}$

$$
C(\%)=\frac{I_{p}}{\left(I_{p}+I_{c}\right)}
$$

\section{Microscopia eletrônica de varredura (MEV)}

A análise por MEV permitiu definir parâmetros e observar a superfície do material e foi realizada no equipamento modelo JEOL-JSM 6460. Para a preparação para análises, as amostras foram dispostas em suporte metálico onde foi introduzido grafite, para favorecer a condutividade do material. ${ }^{27}$ Desse modo, o material não foi submetido à ataque químico, tingimento ou crioultramicrotomia. As ampliações testadas foram 100x, 200x, 500x, 1000x, 1500x e 2000x, com voltagem máxima de $30 \mathrm{kV} .{ }^{15}$

\section{Espectroscopia no infravermelho por transformada de Fourier (FTIR)}

Espectros de FTIR foram adquiridos utilizando o acessório de reflexão total atenuada - ATR (Attenuated Total Reflection), FTIR-ATR, através do equipamento modelo FT/IR-4600 da Jasco, na região do infravermelho $4000-400 \mathrm{~cm}^{1}$, no modo transmitância, com resolução de $4 \mathrm{~cm}^{-1}$ e 32 varreduras. ${ }^{28}$ Todos os espectros foram plotados no programa de computador OiginPro 9.0. As bandas foram observadas com a ferramenta Screen Reader, que permite obter as coordenadas nesse programa. Para permitir a Análise dos Componentes Principais 
(PCA) foram obtidos cinco espectros de cada tipo de amostra e tratadas no programa The Unscrambler.

Durante a análise estatística foi realizado um pré-processamento de dados para eliminar informações que não foram consideradas relevantes. Assim, optou-se por utilizar os intervalos de 2979 a $2810 \mathrm{~cm}^{-1} \mathrm{e}$ de 1739 a $1533 \mathrm{~cm}^{-1}$ dos espectros FTIR-ATR com normalização pelo método de variada normal padrão - SNV (Standard Normal Variate). Tais faixas foram as mais representativas quanto às diferenças entre as amostras devido à formação de bandas.

\section{RESULTADOS E DISCUSSÃO}

\section{Microscopia eletrônica de varredura (MEV)}

Na Figura 2 é possível verificar mudanças significativas de cor e granulometria de acordo com método e tratamento empregado nas esferas de quitosana/PVA (QUI02 e QUI03) quando comparado com a quitosana em pó (QUI01)

Na Figura 3 estão dispostas as imagens obtidas na análise por MEV das amostras QUI01 (Figura 3a e 3b), QUI02 (Figura 3c e 3d) e QUI03 (Figura 3e e 3f). Ao compará-las foi possível verificar
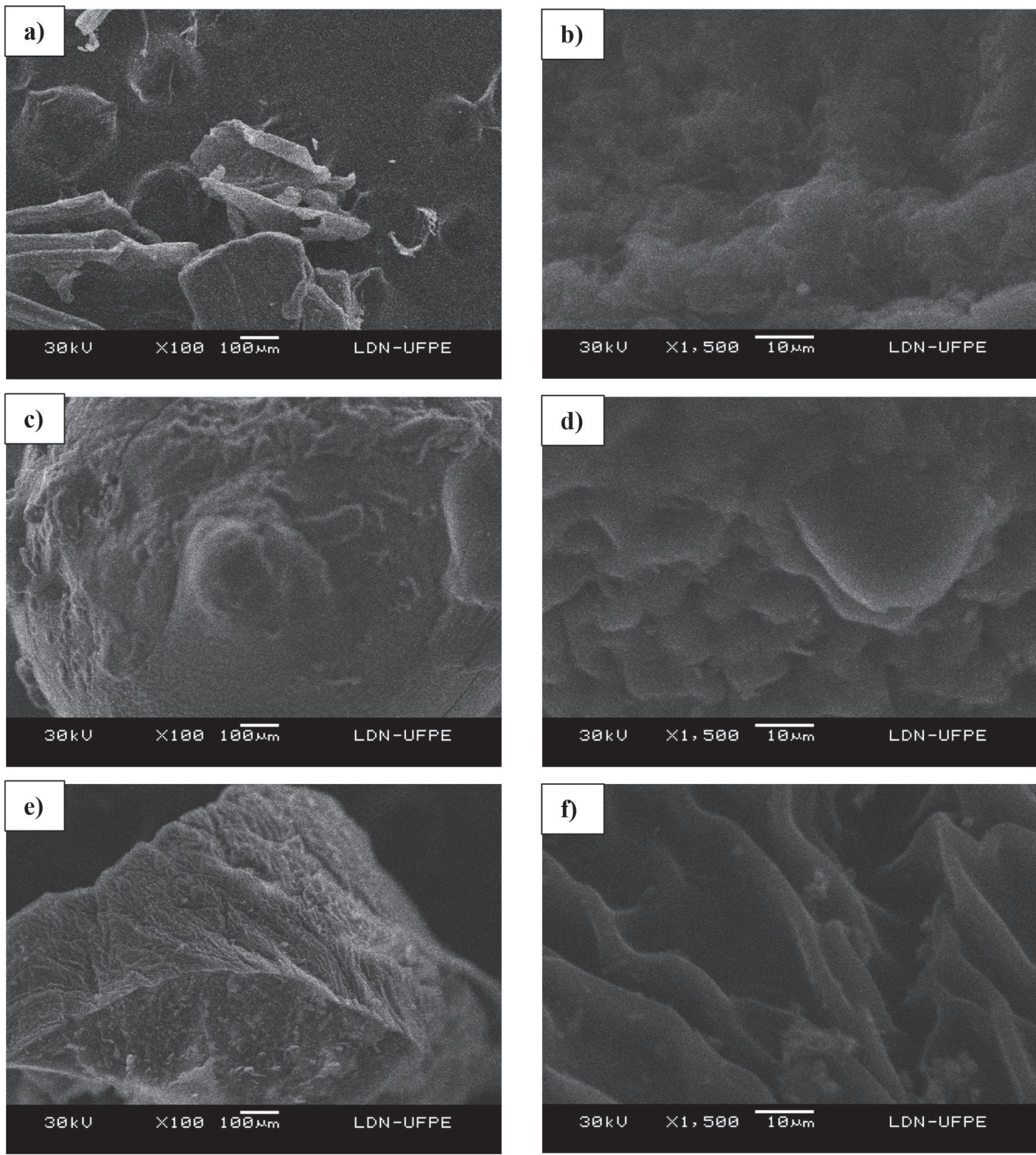

Figura 3. Imagens das amostras obtidas por MEV: quitosana em pó (QUIO1) com aumento de 100x (a) e 1500x (b); esferas de quitosana/PVA secas em estufa (QUI02) com aumento de 100x (c) e 1500x (d); esferas de quitosana/PVA secas em liofilizador (QUI03) com aumento de 100x (e) e 1500x (f) 
comparadas à quitosana em pó. Como a forma desse material inicialmente possuía a superfície relativamente uniforme (Figura 3a, 3c e $3 e$ ), provavelmente a reticulação pode ter aumentado a capacidade de adsorção das esferas em decorrência de sua superfície apresentar rugosidade como mostram as micrografias na Figura 3. Dependendo do tratamento térmico a que foram submetidas, as esferas passaram a adquirir superfícies distintas. Outros estudos já registraram que o agente reticulante pode afetar substancialmente a superfície específica do material reticulado. ${ }^{15}$

Na Figura 3d, foi observada superfície com rugas puntiformes nas esferas da amostra QUI02 que foram secas em estufa, enquanto na superfície das esferas QUI03 (Figura 3f) foram observadas dobras ou rugas transversais, curtas e irregulares. Ao comparar os dois tipos de esferas, as diferenças morfológicas foram principalmente associadas aos diferentes métodos de secagem. Resultados similares foram encontrados para esferas de quitosana reticuladas com tripolifosfato (TPP) e etilenoglicol diglicidil éter (EGDE), secagem a $100{ }^{\circ} \mathrm{C}$, além de quitosana modificada com epicloridrina $(\mathrm{ECH})$, secagem a $90{ }^{\circ} \mathrm{C} .{ }^{15,29}$ Nos referidos trabalhos foram encontradas a morfologia da superfície das amostras não uniforme, mas com presença de macroporos.

Esse atributo pode ser muito interessante para o aumento da aplicabilidade da quitosana no tratamento de resíduos a partir de adsorção, uma vez que a formação de poros ou rugas melhora a estabilidade da quitosana, aumenta a área de superfície e, consequentemente, a eficiência da interação entre adsorvente e adsorbato. ${ }^{29}$ Contudo, é importante obter informações estruturais para entender se houve alterações químicas ou físicas na quitosana reticulada em esferas. ${ }^{30,31}$

\section{Difração de raios-X (DRX)}

Nos difratogramas apresentados na Figura 4 são observados picos característicos da quitosana e que estão de acordo com o plano (110). ${ }^{4,22,23}$ A quitosana (QUI01) apresenta picos cristalinos em $2 \theta$ $=20^{\circ}$ e em torno $2 \theta=10^{\circ}$. Já as amostras QUI02 e QUI03 apresentaram menor intensidade do pico $2 \theta=20^{\circ}$ e desaparecimento do pico $2 \theta=10^{\circ}$, o que demonstra uma mudança na cristalinidade das amostras modificadas.

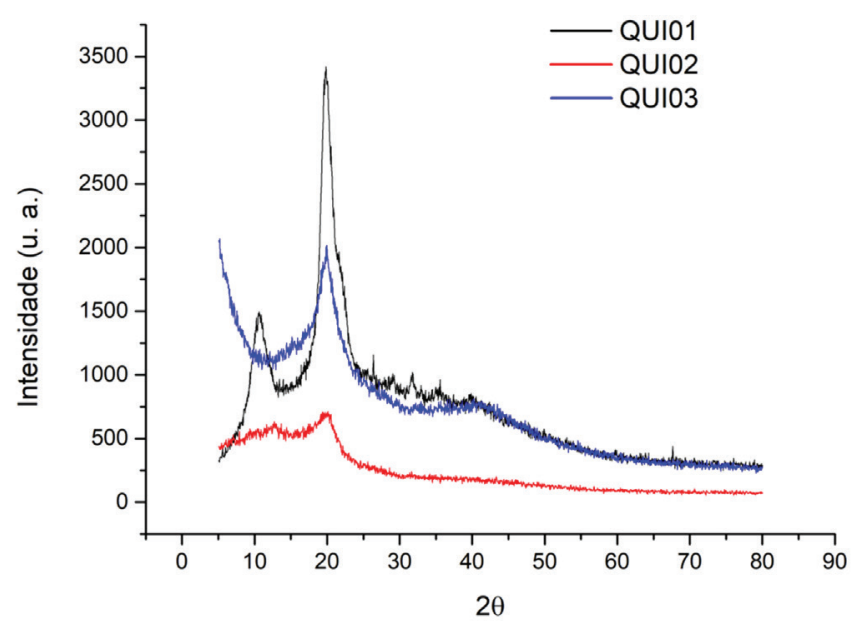

Figura 4. Difratogramas da quitosana (QUIO1), das esferas secas em estufa (QUIO2) e das esferas secas em liofilizador (QUI03)

Os tamanhos de cristais e as fases cristalinas para QUI01, QUI02 e QUI03 encontram-se na Tabela 1. A amostra QUI01 apresentou tamanho de partícula em torno de $20 \mathrm{~nm}$, que corresponde ao valor encontrado na literatura para quitina e quitosana. ${ }^{4}$ Por outro lado, as esferas da amostra QUI03 apresentaram tamanho de partícula em torno de $6 \mathrm{~nm}$, enquanto que para as esferas QUI02 o valor encontrado de aproximadamente $11 \mathrm{~nm}$. A variação do tamanho do cristal está associada ao tratamento térmico a que as esferas foram submetidas. A liofilização permitiu adquirir menor tamanho de cristal quando comparada com a secagem em estufa à $60^{\circ} \mathrm{C}$. Uma possível explicação é que no processo térmico os líquidos da superfície evaporam rapidamente conduzindo a formação de uma camada de material seco na parte externa que faz com a esfera se torne maior do que as esferas que foram produzidas na liofilização.

Tabela 1. Tamanho de cristal e fase cristalina da quitosana (QUI01) e das esferas de quitosana/PVA secas em estufa (QUI02) e liofilizadas (QUI03)

\begin{tabular}{ccccc}
\hline Amostra & $2 \theta\left(^{\circ}\right)$ & $\mathrm{h}(\mathrm{rad})$ & $\begin{array}{c}\text { Tamanho do } \\
\text { cristal }(\mathrm{nm})\end{array}$ & Fase cristalina (\%) \\
\hline QUI01 & 20,231 & 0,035 & 21,27 & 13,11 \\
QUI02 & 20,115 & 0,041 & 11,15 & 3,49 \\
QUI03 & 20,031 & 0,061 & 6,03 & 15,01 \\
\hline
\end{tabular}

A cristalinidade dos materiais produzidos também mostrou diferenças e corroboram com os difratogramas exibidos na Figura 4. As esferas da amostra QUI03 (liofilizador) apresentaram cristalinidade próxima da amostra QUI01, contudo, com uma mudança na organização cristalina que é mostrada pelo desaparecimento do pico $2 \theta=10^{\circ}$ e formação de um pico leve em torno $2 \theta=42^{\circ}$. Esse resultado reforma a influência do tratamento na estrutura cristalina das esferas produzidas.

A cristalinidade das esferas QUI02 se tornou muito menor, ou seja, o aquecimento na preparação das esferas à $60^{\circ} \mathrm{C}$ durante $24 \mathrm{~h}$, resultou em um material com maior fase amorfa muito maior quando comparada com as outras amostras. $\mathrm{O}$ desaparecimento do pico $2 \theta=10^{\circ}$ e o não aparecimento de nenhum outro pico no difratograma (Figura 4) corrobora com esta observação. Esse fato pode ser atribuído à agitação das moléculas sob aquecimento que interferiram no processo de produção e estabilização dos cristais, prejudicando a formação de uma organização cristalina na amostra. Entretanto, essa maior região amorfa da amostra QUI02 poderia resultar em maior abertura e receptividade em processos de adsorção quando comparada com as regiões cristalinas, que possuem estruturas com maior interação entre as moléculas que compõe o material.

\section{Espectroscopia no infravermelho por transformada de Fourier (FTIR)}

Na Figura 5 podem ser visualizados os espectros FTIR-ATR das amostras QUI01, QUI02 e QUI03. O espectro da amostra QUI01 apresentou bandas características da quitosana que já foram registradas em outros estudos. ${ }^{4,18,30}$ Não foram encontradas diferenças significativas nos espectros das amostras QUI02 e QUI03. Para facilitar a identificação das bandas nos espectros, cinco intervalos foram identificados e atribuição das bancas em cada intervalo estão apresentadas na Tabela 2.

No primeiro intervalo, a banda em na região de $3595 \mathrm{~cm}^{-1}$ são atribuídas as vibrações das ligações $\mathrm{O}-\mathrm{H}$ do grupo hidroximetil $\left(\mathrm{CH}_{3} \mathrm{OH}\right)$ observadas nas molécula de quitosana. ${ }^{18,32,33}$ No terceiro intervalo foram verificadas as ligações do tipo $\mathrm{C}-\mathrm{C}\left(1650 \mathrm{~cm}^{-1}\right)$ e $\mathrm{C}-\mathrm{O}\left(1639 \mathrm{~cm}^{-1}\right)$ relacionadas com o grupo de aminoacetilados. Nesse mesmo intervalo podemos destacar o pico próximo a $1561 \mathrm{~cm}^{-1}$ equivalentes às ligações $\mathrm{C}-\mathrm{N}-\mathrm{H}$, também presentes nos grupos aminoacetilados. ${ }^{4,30}$

No quarto intervalo correspondente foram encontradas bandas próximas a $1453 \mathrm{~cm}^{-1}$ referente ao grupo $-\mathrm{NH}_{2}, 1371 \mathrm{~cm}^{-1}$ referente 


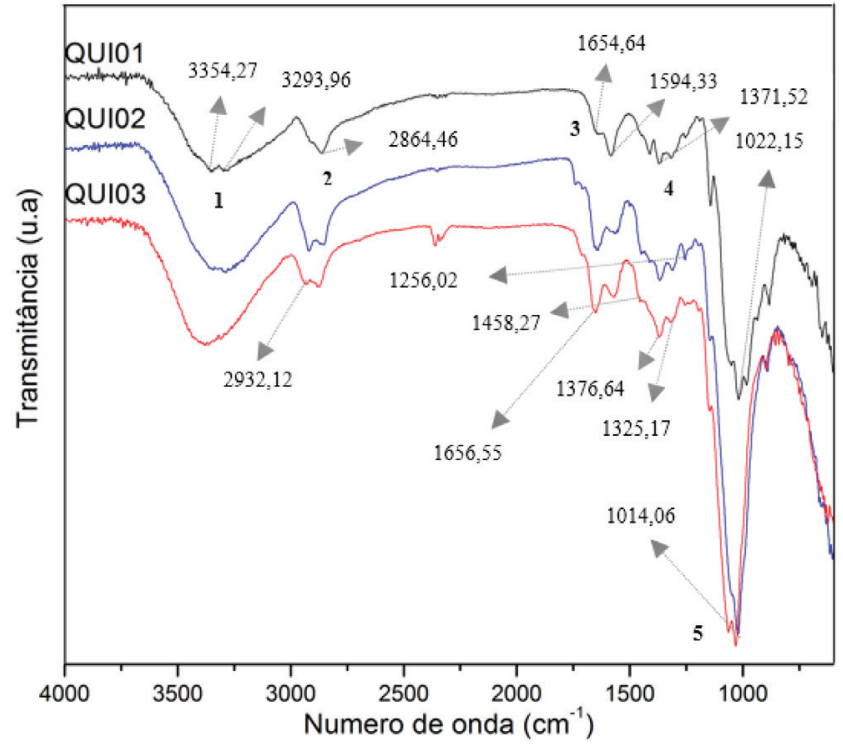

Figura 5. Espectros FTIR-ATR da quitosana em pó - QUIO1, das esferas QUIO2 e QUIO3

Tabela 2. Atribuição das bandas encontradas nos espectros de FTIR-ATR das amostras QUI01, QUI02 e QUI03 $3,4,13,30,32,33$

\begin{tabular}{|c|c|c|}
\hline Intervalo & $\begin{array}{l}\text { Intervalo do Número } \\
\text { de Onda }\left(\mathrm{cm}^{-1}\right)\end{array}$ & Tipo de Ligação \\
\hline 1 & $3595-3083$ & $\begin{array}{l}\mathrm{O}-\mathrm{H} \\
\mathrm{N}-\mathrm{H}\end{array}$ \\
\hline 2 & $2959-2827$ & $\mathrm{C}-\mathrm{H}$ \\
\hline 3 & $1757-1534$ & $\begin{array}{c}\mathrm{C}=\mathrm{C} \\
\mathrm{C}=\mathrm{O} \\
\mathrm{C}-\mathrm{N}-\mathrm{H}\end{array}$ \\
\hline 4 & $1474-1223$ & $\begin{array}{l}\mathrm{N}-\mathrm{H} \\
\mathrm{C}-\mathrm{H} \\
\mathrm{C}-\mathrm{O} \\
\mathrm{C}-\mathrm{N}\end{array}$ \\
\hline 5 & $1176-880$ & $\begin{array}{l}\mathrm{C}-\mathrm{O} \\
\mathrm{C}-\mathrm{C}\end{array}$ \\
\hline
\end{tabular}

ao grupo $-\mathrm{CH}_{3}, 1316 \mathrm{~cm}^{-1}$ referente ao grupo $\mathrm{C}-\mathrm{O}$ e $1260 \mathrm{~cm}^{-1}$ referente à ligação $\mathrm{C}-\mathrm{N}^{4,13,33} \mathrm{O}$ quinto e último intervalo foi associado às vibrações das ligações do tipo C-O ou C-C entre 1300 e $800 \mathrm{~cm}^{-1}$. $^{30}$ As amostras também apresentaram bandas em $1027 \mathrm{~cm}^{-1}$, que corresponde à vibração das ligação $\mathrm{C}-\mathrm{O}$ (entre 1100 e $1000 \mathrm{~cm}^{-1}$ ), assim como bandas atribuídas às vibrações das ligações das estruturas de polissacarídeos entre 880 e $1130 \mathrm{~cm}^{-1} .3,4$

Por outro lado, as bandas relativas à estrutura do PVA devem ter ficado sobrepostas nos espectros relativos às amostras QUI02 e QUI03. Em espectros do PVA são encontradas bandas características de grupos similares aos encontrados na quitosana que justificam a sobreposição. $^{32}$

Deslocamentos de bandas nos espectros podem estar associadas a um fenômeno físico de atração e repulsão entre as moléculas, enquanto a variação de intensidade e o alargamento das bandas estariam relacionados com alterações em parâmetros físicos, como momento dipolar, que resultaria em mudanças nas características vibracionais. ${ }^{33}$ A ausência de mudanças significativas como surgimento de bandas confirmaria a incorporação dos agentes reticuladores. ${ }^{34,35} \mathrm{O}$ fato de não serem encontradas de forma visível modificações nas bandas dos espectros apresentados na Figura 5 podem guardar mudanças intrínsecas que são devidamente identificadas através de ferramentais quimiométricos, como uma análise de PCA, que permite o reconhecimento de padrões.

Na Figura 6 são mostrados os resultados de PCA para as amostras na região do segundo intervalo que corresponderam à região de ligação do tipo C-H de $\mathrm{CH}_{2} \mathrm{OH}$ (Tabela 2). Na Figura 6a foram plotadas as correlações das amostras com as componentes principais PC1 e PC2 e na Figura 6b o gráfico de cargas mostra onde há diferenças entre as amostras no intervalo do espectro analisado. Para facilitar a compreensão dos resultados, os gráficos de $\mathrm{PC} 1 \mathrm{E}$ PC2 foram identificadas em quadrante de I a IV. No quadrante I ficaram localizadas as esferas QUI03, enquanto as amostras QUI01 se agruparam no quadrante IV. Por outro lado, as esferas QUI02 se agruparam nos quadrantes II e III, ou seja, foram apresentadas as amostras com separação de grupos na dimensão da PC2. Esses agrupamentos indicaram diferenças nas amostras, mesmo que não fortemente evidenciadas nos espectros FTIR-ATR da Figura 5. Essas diferenças foram provavelmente associadas às modificações na quitosana por meio da preparação das esferas e pelos tratamentos térmicos às quais foram submetidas. As diferenças encontradas na análise de PCA no intervalo estudado foram evidenciadas na banda $2932 \mathrm{~cm}^{-1}$, referente ao estiramento da ligação C-H, e mostra onde as principais interações ou modificações aconteceram nas amostras.

Para o segundo intervalo estudado, $1739 \mathrm{e} 1533 \mathrm{~cm}^{-1}$ (Tabela 2), as correlações das amostras com as componentes PC1 e PC2 (Figura 7a) também mostraram formação de grupos. No primeiro quadrante se agruparam as amostras QUI01, no segundo, quadrante se agruparam as esferas QUI02, e nos terceiro e quarto quadrantes se agruparam as amostras QUI03. Nesse intervalo foram apresentadas as amostras com separação de grupos na dimensão da PC1.

O gráfico de cargas (Figura 7b) mostra as regiões de maiores diferenças nos espectros FTIR nas regiões de $1690 \mathrm{~cm}^{-1}$ e próximo à $1560 \mathrm{~cm}^{-1}$ relacionados com as vibrações das ligações do tipo $\mathrm{C}=\mathrm{O}$ e $\mathrm{C}-\mathrm{N}-\mathrm{H}$, respectivamente. $\mathrm{O}$ tratamento térmico pode influenciar na vibração das ligações entre carbonos e resultar na despolimerização do material. ${ }^{35}$ Além disso, a intensidade da banda $1590 \mathrm{~cm}^{-1}$ pode estar relacionado à ligações $\mathrm{C}-\mathrm{N}-\mathrm{H}$ que seria associada ao grupo amina da quitosana e o glutaraldeído durante a reticulação. ${ }^{36}$

\section{CONCLUSÕES}

As esferas de quitosana/PVA preparadas por meio de reticulação seguidas de diferentes tratamentos térmicos distinguiram-se em tamanho de partículas e fase amorfa. As esferas de quitosana/PVA tratadas por liofilização (QUI03) demonstraram menores tamanhos de cristal, enquanto o tratamento térmico em estufa (QUI02) resultou em menor fase cristalina, ou seja, maior região amorfa. Essa variação pode influenciar na eficiência de reações devido à superfície de contato e a presença de sítios ativos, relacionados com a maior fase amorfa. Presença de rugas foram confirmadas pelas micrografias, indicando possíveis propriedades físicas e químicas diferenciadas para as esferas reticuladas tratadas termicamente. Além disso, os espetros FTIR mostraram semelhanças químicas, contudo, mudanças físicas no que tange a interação entre moléculas puderam ocorrer, principalmente se considerar os resultados da quimiometria. A partir das análises, as diferenças observadas estiveram relacionadas com o processo de fabricação das esferas de quitosana/PVA e foram evidenciadas nas ligações moleculares, enquanto a estrutura química permaneceu semelhante mesmo após as modificações. Essas modificações influenciaram nas propriedades e características do biopolímero, em que o tamanho de cristal, a porcentagem de fase amorfa e a presença de rugas podem resultar em melhor receptividade para reações químicas e maior capacidade para aplicações baseadas na adsorção e captação de contaminantes em meio aquoso. 

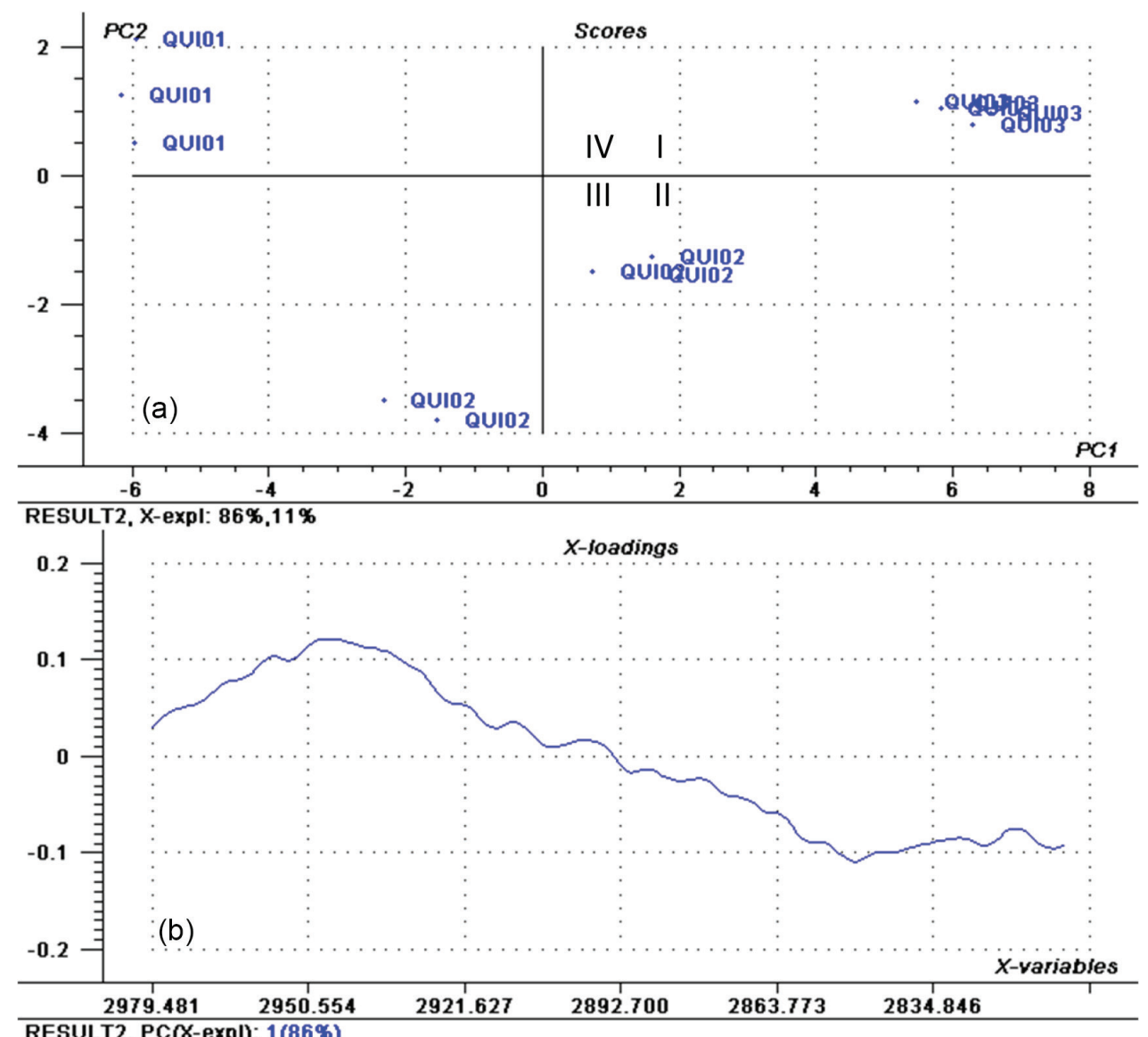

RESULT2, PC(X-expl): 1(86\%)

Figura 6. Análise de Componente Principal: a) gráfico de dispersão das componentes principais PC1 e PC2 no intervalo dos espectros FTIR entre 2979 e $\left.2810 \mathrm{~cm}^{-1} \mathrm{e} \mathrm{b}\right)$ gráfico das cargas da componente principal

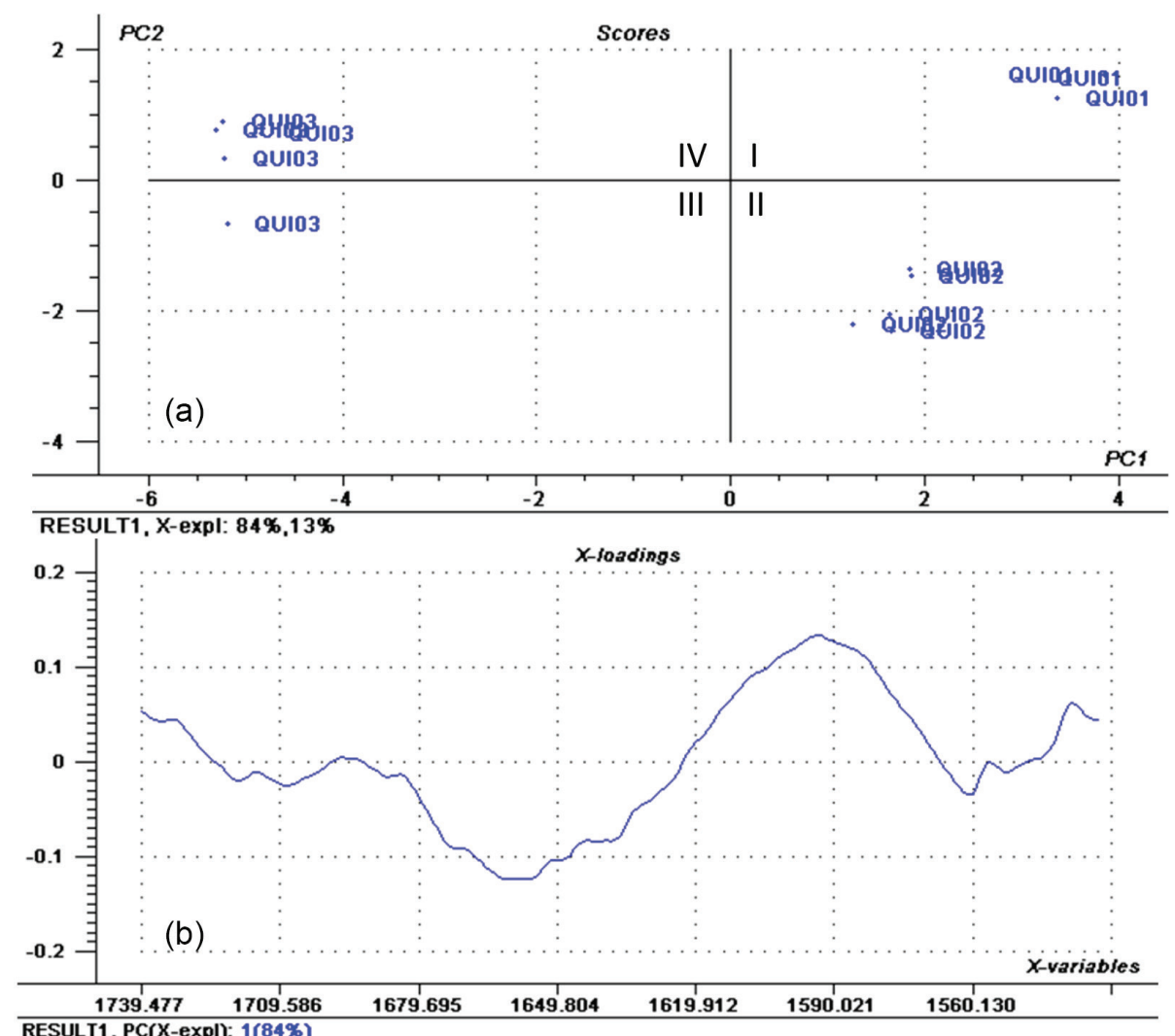

Figura 7. Análise de Componente Principal: a) gráfico de dispersão das componentes principais PC1 e PC2 no intervalo dos espectros FTIR entre 1739 e $1533 \mathrm{~cm}^{-1}$ e b) gráfico das cargas da componente principal 


\section{AGRADECIMENTOS}

Os autores agradecem à CAPES, à Área de Pesquisa, Desenvolvimento, Inovação e Ensino do CRCNNE, ao Laboratório Multiusuário de Nanotecnologia do CETENE, ao Núcleo de Inovação Tecnológica do IFPE, ao Laboratório de Polímeros e Nanoestruturas e Laboratório de Dispositivos e Nanoestruturas da UFPE, pelo suporte para o desenvolvimento da presente pesquisa.

\section{REFERENCIAS}

1. White, S. A.; Farina, P. R.; Fulton, I.; Appl. Environ. Microbiol. 1979 , 38,323 .

2. Craveiro, A. A.; Craveiro, A. C.; Queiroz, D. C.; Quitosana: A Fibra do Futuro, PADETEC: Fortaleza, 2004.

3. Castelló, M. E.; Anbinder, P. S.; Amalvy, J. I.; Peruzzo, P. J.; MRS Adv. 2018, 3, 3601.

4. Gachhi, D. B.; Hungund, B. S.; J. Appl. Pharm. Sci. 2018, 8, 116.

5. Modi, M. K.; Pattanaik, P.; Dash, N.; Subramanian, S.; Int. J. Pharm. Sci. Rev. Res. 2015, 34, 122.

6. Aguilar, A.; Zein, N.; Harmouch, E.; Hafdi, B.; Bornert, F.; Offner, D.; Clauss, F.; Fioretti, F.; Huck, O.; Jessel, A. B.; Hua, G.; Molecules, MDPI 2019, 24, 3009.

7. Sahu, S.; Kabra, P.; Choudhary, E.; International Journal of Dental Science and Innovative Research 2019, 2, 6.

8. Tosun, S.; J. Adv. Oral Res. 2019, 10, 161.

9. Soygun, K.; Soygun, A.; Dogan, M. C.; Microsc. Res. Tech. (2019), doi: 10.1002/jemt.23382.

10. Yang, Y.; Xing, R.; Liu, S.; Qin, Y.; Li, K.; Yu, H.; Li, P.; Carbohyd. Polym. (2020), doi: 10.1016/j.carbpol.2019.115423.

11. Lichtfouse, E.; Crini, N. M.; Fourmentin, M.; Zemmouri, H.; Nascimento, I. O. C.; Queiroz, L. M.; Tadza, M. Y. M.; Corrales, L. A. P.; Pei, H.; Wilson, L. D.; Crini, G.; Environ. Chem. Lett. 2019, 128, 1.

12. Harutyunyan, L. R.; Harutyunyan, R. S.; Gabrielyan, G. A.; Lasareva, E. V.; Colloids Surf. (2019), doi: 10.1016/j.colsurfa.2019.123622.

13. González, P. A.; Justo, J. A. Z.; López, A. S.; Martínez, G. R. V.; López, J. A. B.; Diosdado, A. M.; Hernández, M. I.; Nanoscale Res. Lett. 2019, $14,258$.

14. Kaygusuz, H.; Torlak, E.; Evingür, G. A.; Özen, I.; Klitzing, R.; Erim, F. B.; Int. J. Biol. Macromol. 2017, 105, 1161.

15. Nitsae, M.; Madjid, A.; Hakim, L.; Sabarudin, A.; Chem. Chem. Technol. 2016, 10, 105 .

16. Iglesias, R. B.; Coronilla, R.; Concheiro, A.; Lorenzo, C. A.; Eur. J. Pharm. Sci. 2004, 24, 77.
17. Kulkarni, V. H.; Kulkarni, P. V.; Keshavayya, J.; J. Appl. Polym. Sci. 2007, 103, 211.

18. Ali, M.; Gherissi, A.; International Journal of Mechanical \& Mechatronics Engineering 2017, 17, 15.

19. Callister, W. D.; Ciência e Engenharia de Materiais: Uma Introdução, LTC - Livros Técnicos e Científicos Editora S. A.: Rio de Janeiro, 2002.

20. Onofre-Cordeiro, N. A.; Silva, Y. E. O.; Solidonio, E. G.; de Sena, K. X. F. R.; Silva, W. E.; Santos, B. S.; Aquino, K. A. S.; Lima, C. S. A.; Yara, R.; Int. J. Biol. Macromol. 2018, 119, 701.

21. Sousa, G. D.; Kishishita, J.; Aquino, K. A. S.; Presgrave, O. A. F.; Leal, L. B.; Santana, D. P.; AAPS PharmSciTech. 2017, 18, 1833.

22. Yusof, N. A. A.; Zain, N. M.; Pauzi, N.; Bull. Chem. React. Eng. Catal. 2019, 14, 450 .

23. Singh, G.; Faruk, A.; Bedi, P. M. S. J.; Drug Delivery Ther. 2018, 8, 111.

24. Muniz, F. T. L.; Miranda, M. A. R.; Santos, C. M.; Sasaki, J. M.; Acta Crystallogr. 2016, 72, 385.

25. Ruland, W.; Acta Crystallogr. 1961, 14, 1180.

26. https://www.originlab.com/pdfs/Tutorials.pdf, acessada em agosto de 2020.

27. Dedavid, B. A.; Gomes, C. I.; Machado, G.; Microscopia Eletrônica de Varredura. Aplicações e Preparação de Amostras. Materiais Poliméricos, Metálicos e Semicondutores, EDIPUCRS: Porto Alegre, 2007.

28. Silva, L. A.; Almeida, T. M. B.; Teixeira, R. V.; Araujo, E. S.; Aquino, K. A. S.; Mater. Res. 2017, 20, 709.

29. Masykur, A.; Wibowo, A. H.; Martini, T.; Laras, K. C.; J. Phys. Conf. Ser. 2018, 1028, 1.

30. Silverstein, R. M.; Webster, F. X.; Kiemle, D. J.; Spectrometric Identification of Organic Compounds, John Wiley \& Sons: United States of America, 2005.

31. Hanumantharaju, H. G.; Prashanth, K. P.; Int. J. Eng. Sci. Res. 2019, 26, 119.

32. Mishra, S. P.; Sarkar, U.; Taraphder, S.; Datta, S.; Swain, P.; Saikhom, R. Panda, S.; Laishram, M.; International Journal of Livestock Research 2017, 7, 60 .

33. Ibrahim, M.; Osman, O.; Mahmoud, A. A. J.; Comput. Theor. Nanosci. 2011, 8, 117.

34. Costa Jr., E.; Mansur, H. S.; Quim. Nova 2008, 31, 1460.

35. Arantes, M. K.; Kugelmeier, C. L.; Cardoso-Filho, L.; Monteiro, M. R.; Oliveira, C. R.; Alves, H. J.; Polym. Eng. Sci. 2014, 55, 1969.

36. Beppu, M. M.; Vieira, R. S.; Aimoli, C. G.; Santana, C. C.; J. Membr. Sci. 2007, 301, 126. 